\title{
Video evidence that parenting methods predict which infants develop long night-time sleep periods by three months of age
}

\author{
Ian St James-Roberts ${ }^{a}$, Marion Roberts, Kimberly Hovish and Charlie Owen \\ Thomas Coram Research Unit, UCL Institute of Education, University College London, London, UK
}

\begin{abstract}
Aim: To examine two hypotheses about the longitudinal relationship between night-time parenting behaviours in the first few postnatal weeks and infant night-time sleep-waking at five weeks, three months and six months of age in normal London home environments. Background: Most western infants develop long night-time sleep periods by four months of age. However, around $20-30 \%$ of infants in many countries continue to sleep for short periods and cry out on waking in the night: the most common type of infant sleep behaviour problem. Preventive interventions may help families and improve services. There is evidence that 'limit-setting' parenting, which is common in western cultures, supports the development of settled infant night-time behaviour. However, this evidence has been challenged. The present study measures three components of limit-setting parenting (response delay, feeding interval, settling method), examines their stability, and assesses the predictive relationship between each of them and infant sleep-waking behaviours. Methods: Longitudinal observations comparing a General-Community $(n=101)$ group and subgroups with a Bed-Sharing ( $n=19)$ group on infra-red video, diary and questionnaire measures of parenting behaviours and infant feeding and sleep-waking at night. Findings: Bed-Sharing parenting was highly infant-cued and stable. General-Community parenting involved more limit-setting, but was less stable, than Bed-Sharing parenting. One element of GeneralCommunity parenting - consistently introducing a short interval before feeding - was associated with the development of longer infant night-time feed intervals and longer day-time feeds at five weeks, compared with other General-Community and Bed-Sharing infants. Twice as many General-Community infants whose parents introduced these short intervals before feeding in the early weeks slept for long night-time periods at three months of age on both video and parent-report measures, compared with other General-Community and Bed-Sharing infants. The findings' implications for our understanding of infant sleepwaking development, parenting programmes, and for practice and research, are discussed.
\end{abstract}

Key words: infant crying; infant sleeping; parenting

Received 6 March 2016; revised 15 August 2016; accepted 20 November 2016;

first published online 28 December 2016

Waking and crying out at night is reported in around $20-30 \%$ of infants in many countries

Correspondence to: Emeritus Professor Ian St James-Roberts, Thomas Coram Research Unit, UCL Institute of Education, University College London, 27/28 Woburn Square, London WC1H 0AA. Email: i.stjamesroberts@ucl.ac.uk

${ }^{\text {a }}$ Ian St James-Roberts was Professor of Child Psychology when the study was carried out.
(Sadeh and Sivan, 2009; Mindell et al., 2010), making this the earliest and most common type of infant sleep behaviour problem. Interventions which prevent this problem may help many families and improve services.

Building on evidence that most western infants develop long sleep periods at night by four months of age (Moore and Ucko, 1957; Anders et al., 1992; Henderson et al., 2010), four

(C) Cambridge University Press 2016. This is an Open Access article, distributed under the terms of the Creative Commons Attribution licence (http://creativecommons.org/licenses/by/4.0/), which permits unrestricted re-use, distribution, and reproduction in any medium, provided the original work is properly cited. 
randomised-controlled trials (RCTs) showed that 'limit-setting' parenting increased the number of infants with long night-time sleep periods, and reduced night-time 'signalling' (crying out), during this key stage for sleep-waking development (Wolfson et al., 1992; Pinilla and Birch, 1993; St James-Roberts et al., 2001; Symon et al., 2005). 'Limit-setting' parenting is common in western societies, employing routines and delayed responding to encourage infants to develop autonomous settling (Jenni and O'Connor, 2005; St James-Roberts et al., 2006). In contrast, 'infantcued' parenting, which includes high proximity, rapid responses and, in some cases, bed-sharing, is associated with persistent infant signalling at night (St James-Roberts et al., 2006; Sadeh et al., 2010; Hysing et al., 2014). Importantly, limit-setting parenting increased breast-fed infants' night-time sleep lengths by four months without affecting weight gain (Pinilla and Birch, 1993; St James-Roberts et al., 2001), indicating compatibility with breast-feeding.

Given this evidence, it is puzzling that two attempts to apply these findings to community health services did not increase the numbers of infants with long night-time sleep periods (Stremler et al., 2013; Hiscock et al., 2014). This may be because limit-setting parenting was common in the communities involved so that intervention and control groups were too similar, as Stremler et al. (2013) argue. However, a recent report has concluded that limit-setting parenting does not support sleep-waking development and risks increasing infant distress (Douglas and Hill, 2013).

Analyses in a linked report examine whether limit-setting parenting is associated with infant distress at night (St James-Roberts et al., 2016). In summary, infants in the limit-setting group had around $30 \mathrm{~min}$ more distress per night at two weeks $(2 \mathrm{~W})$, reducing to $12-13 \mathrm{~min}$ per night by three months (3M) of age, compared with BedSharing infants. However, excluding Bed-Sharing cases, differences in infant distress between General-Community subgroups adopting limitsetting versus infant-cued parenting were not large or statistically significant at any age.

The present report addresses the review's other query by examining longitudinal relationships between limit-setting parenting and infant nighttime sleeping in the first $3 \mathrm{M}$ of age in the same infants and parents. As well as parental reports, we used infra-red video recording methods developed by Anders et al. and proven valid for observation of night-time behaviours at home (Anders and Keener, 1985; Goodlin-Jones et al., 2001; Sitnick et al., 2008). Analyses were guided by two hypotheses as to how limit-setting parenting might encourage the development of infant sleep-waking at night:

(1) Our primary hypothesis was that delayed parenting response at prior ages would reduce infant night-time signalling at $3 \mathrm{M}$ and $6 \mathrm{M}$ of age. Derived from learning theory and sleep problem treatment studies, this hypothesis posits that immediate parental response maintains infant night-time signalling until later ages, while delayed response extinguishes it. Because some parents vary their care strategies during the postnatal period (GoodlinJones et al., 2001), we assessed parenting stability, versus instability, in predicting infant sleep-waking.

(2) Our secondary hypothesis was that implementing an interval between infant waking and feeding (rather than feeding immediately) would predict prolonged infant sleep periods, and reduced signalling, at night. This 'feeding interval' hypothesis was based on Wright's (1993) observation that breast-fed infants increase morning feeds to be larger than other feeds by two months of age, presumably in response to their night-time fast. Further, it reflected limit-setting guidelines that parents should introduce short intervals between infant waking and feeding, for example for nappy (diaper) changes, to break the association (Pinilla and Birch, 1993; St James-Roberts et al., 2001). We hypothesised that infants whose parents implemented an interval between infant waking and feeding would develop longer night-time inter-feed intervals and longer day-time feeds at five weeks $(5 \mathrm{~W})$, leading to longer night-time sleep periods at $3 \mathrm{M}$ and $6 \mathrm{M}$ of age.

\section{Methods}

The study received Riverside Medical Research Ethics Committee approval (REC 09/H0706/11). 


\section{Participants}

Two groups of mothers and breast-feeding singleton infants living within the M25 motorway around London, UK were recruited. The 'General-Community' group $(n=101)$ was enrolled in postnatal wards of a general community maternity hospital. We excluded multiple births, infants with birth weight $<2500 \mathrm{~g}$, admitted to special care, who medical staff considered unwell, and mothers with limited English. Otherwise, mothers were approached consecutively, introduced to the study, and asked to allow a telephone call to explain the research fully after returning home. Mothers who gave written informed consent completed the newborn Infant Sleep and Feeding Arrangements Questionnaire (ISFAQ), described below, when infants were $<48 \mathrm{~h}$ old.

For comparison, we recruited a group of mothers who planned to adopt highly infant-cued parenting. Prior studies found that mothers who intend to bed-share with their babies are likely to adopt infantcued parenting behaviours in general (St JamesRoberts et al., 2006; Hysing et al., 2014). Mothers included in this study's planned Bed-Sharing group met the recruitment criteria of the General-Community group but intended to bedshare with their babies most of the night (defined as $\geqslant 90 \%$ of the night, $\geqslant 5$ nights/week based on the mothers' ISFAQ answers: see Table 1). Only one mother approached in the maternity hospital met these criteria; most (18 of 19) were recruited during pregnancy via parenting networks. They gave written informed consent and completed the ISFAQ before or within $48 \mathrm{~h}$ of their baby's birth. This group's size was small despite 18 months of recruitment, possibly because this coincided with medical guidance that bed-sharing is unsafe. Table 1 in St James-Roberts et al. (2016) provides detailed recruitment, participant demographic and missing data for General-Community and Bed-Sharing groups.

\section{Newborn assessments}

Following earlier studies (Morrell, 1999; St James-Roberts et al., 2001; Tikotzky and Sadeh, 2009) the newborn ISFAQ was constructed to provide a brief screen for the mothers' intended parenting strategies at home. Mothers answered seven questions by ticking boxes or inserting figures. Table 1 gives the wording of the ISFAQ items, including those used to assess response delay and feeding interval (items 6 and 7). Other items measured whether parents intended to use evening settling routines (item 4) and whether they planned to settle their baby to sleep at a regular time or when tired ('settling method', item 5).

\section{$2 \mathrm{~W}$ assessments}

Full written informed consent was obtained at a home visit when infants were 10-14 days old. Parents provided demographic information and the ISFAQ was repeated, re-worded to refer to current parenting practices. Researchers explained the Baby Day Diary (Diary) and asked parents to keep this for $3 \times 24 \mathrm{~h}$ days. The Diary is a validated, real-time, parent-report measure of infant sleep, fuss/crying, and awake-settled behaviour (Barr et al., 1988; 2005).

\section{$5 \mathrm{~W}$ assessments}

ISFAQ current parenting and Diary measures were repeated. Following prior studies (GoodlinJones et al., 2001; Ball, 2007; Sitnick et al., 2008) researchers installed a self-focussing digital infrared video camera (Sony HDR-XR200VE) on a tripod directed at the infant's night-time sleep location, allowing up to $13 \mathrm{~h}$ of continuous recording. Parents were instructed in camera use and asked to switch it on when they began settling their infant to sleep at night and off the following morning. They were asked to follow their usual night-time habits. Parents decided when the camera was switched on at night and off the following morning and could switch the equipment off at other times if they chose to do so. The video was checked by researchers the following day and, if technical problems had arisen, one further attempt was made to obtain a recording.

\section{M assessments}

The ISFAQ, Diary and video measures were repeated and parents completed the Brief Infant Sleep Questionnaire (BISQ, Sadeh, 2004). BISQ items include the average number of times/night parents detected infant waking, a widely used outcome measure of infant sleep-waking adopted here. Sleeping continuously for $\geqslant 5 \mathrm{~h} /$ night is the criterion for settled night-time behaviour used 
Table 1 The Infant Sleep and Feeding Arrangements Questionnaire (ISFAQ) items

\begin{tabular}{|c|c|}
\hline Items $^{a}$ & Possible answers \\
\hline $\begin{array}{l}\text { 1. How are you planning to feed (currently } \\
\text { feeding) your baby? }\end{array}$ & $\begin{array}{l}\text { Breast only } \\
\text { Breast + expressed breast milk } \\
\text { Breast + formula milk } \\
\text { Formula only }\end{array}$ \\
\hline 2. Where will (does) baby usually sleep at night? & $\begin{array}{l}\text { In parents' bed } \\
\text { Mattress/futon next to parents' bed } \\
\text { Cot next to parents' bed } \\
\text { Cot elsewhere in parents' bedroom } \\
\text { Cot in sibling's room } \\
\text { Cot in separate room }\end{array}$ \\
\hline 3. Will (does) baby ever sleep in bed with you? & $\begin{array}{l}\text { No } \\
\text { Yes }^{b}\end{array}$ \\
\hline $\begin{array}{l}\text { 4. Are you planning to use (currently using) routines each } \\
\text { evening - such as bathing at a regular time - to } \\
\text { help baby to settle at night? }\end{array}$ & $\begin{array}{l}\text { No } \\
\text { Yes } \\
\text { Not thought about it }\end{array}$ \\
\hline $\begin{array}{l}\text { 5. Do you think it is best to put your baby down to } \\
\text { sleep at the same time each night, or when he/she } \\
\text { is tired and starts to fall asleep? }\end{array}$ & $\begin{array}{l}\text { Always settle when tired } \\
\text { Almost always when tired } \\
\text { Usually when tired } \\
\text { Sometimes tired/sometimes at a regular time } \\
\text { Usually same time } \\
\text { Almost always same time } \\
\text { Always same time }\end{array}$ \\
\hline $\begin{array}{l}\text { 6. If baby wakes in the night, how many minutes are you } \\
\text { likely to leave him/her to cry before you pick him/her up? } \\
\text { (Record } 0 \mathrm{~min} \text { if you never leave him/her to cry) }\end{array}$ & Minutes \\
\hline $\begin{array}{l}\text { 7. If baby wakes in the night, how many minutes will (does) } \\
\text { it take before you feed him/her? (Record } 0 \text { min if you always } \\
\text { feed immediately) }\end{array}$ & Minutes \\
\hline
\end{tabular}

${ }^{a}$ Newborn period wording asked for parents' plans after they got home; wording at later ages (in brackets) asked about current practices.

${ }^{b}$ If yes, mothers answered two subsidiary questions:

(a) when baby sleeps in your bed with you at night, will this usually be (is this usually):

(i) all night (90-100\% of the night); (ii) most but not all of the night; (iii) for short periods (eg, just for feeding);

(iv) other (please specify)

(b) If you selected (i) or (ii) above, how many nights per week will this (does this) usually happen? (circle one): number of nights per week $1,2,3,4,5,6,7$.

in previous studies of this age-group (Moore and Ucko, 1957; Pinilla and Birch, 1993; St James-Roberts et al., 2001). To assess this, we asked: In the last week, how many nights has your baby slept continuously for 5 hours or longer? Answers were zero to seven nights. Because of extensive assistance with data collection, parents received high street shopping vouchers value $£ 100$ on returning the $3 \mathrm{M}$ data.

\section{Six-month (6M) follow-up}

After a telephone follow-up, the ISFAQ, BISQ and 5-h sleep period measures were repeated and returned by mail.

\section{Data coding and analysis}

The data were computer coded in Excel spreadsheets by researchers using written manuals and trained to $\geqslant 90 \%$ reliability. Parental report data were coded blinded. Video coders cannot remain blinded, but video and parental report data were coded by different researchers. The Excel data were exported to SPSS 22 (IBM, 2013) for analysis.

The start time and end time of each diary behaviour period (sleeping, awake content, fussing or crying, feeding) was coded, allowing measures of the timing, duration and frequency of each behaviour. Following earlier findings, the diary night-time was defined as $7 \mathrm{pm}$ to $7 \mathrm{am}$, day-time 
from 7am to 7pm (St James-Roberts et al., 2001). One night of video recording per infant at each age was coded. Video coding rules were based on Anders' methods and conventional definitions of infant behaviour states (Anders and Keener, 1985; Goodlin-Jones et al., 2001). Detailed descriptions are in St James-Roberts et al. (2015). In summary, the videos were coded to identify five behaviour period types: awake; sleep; indeterminate; out of view; video turned off. Within each awake and sleep period, the times, frequencies and durations of infant behaviours (sleep, drowsy, awake content, fuss/crying, feeding), of 'direct parental contact' (touching, holding or speaking to an infant) and of 'checking' (approach to an infant without direct contact) were coded. To confirm reliability, 20 videos and 20 diaries were duplicate-coded by independent coders. Video between-coder Pearson correlations ranged from 0.862 to 1.00 . Diary overall coding agreement was 0.998 .

\section{Data analysis methods}

Detailed ISFAQ, video and Diary data at each age within the first $3 \mathrm{M}$ are reported in a linked publication (St James-Roberts et al., 2016). In summary, compared with Bed-Sharing parents, General-Community parents were much more likely to delay responding to infant crying, to introduce an interval before feeding, and to settle infants for their night-time sleep at a regular time, at each age. These findings were replicated across methods and were the main parenting differences between these two groups. Building on these findings, analyses here examine whether each of these three elements of limit-setting versus infantcued parenting predicts infant night-time sleepwaking at $3 \mathrm{M}$ and $6 \mathrm{M}$.

Before examining the hypothesised predictions, parenting stability needed to be taken into account. One reason for this, reflecting GoodlinJones et al.'s (2001) findings, is that we expected the early postnatal weeks would be a transitional period for many parents as they tried out and adapted their methods of care. That is, some parents would not implement their planned forms of care, or would change them over time. Second, the limit-setting parenting literature implies that infants learn self-regulatory strategies which help them to sleep for long periods at night (Henderson et al., 2010; St James-Roberts et al., 2015). If so, consistent environmental cues may be important in supporting development of day: night differences in sleep-waking and extended night-time sleep periods. An inconsistent parenting environment might hamper this learning.

Following these rationales, data analysis involved three steps. First, employing a mediansplit method, the General-Community median newborn ISFAQ scores were used to divide parenting into infant-cued (above the median) versus limit-setting (below the median) categories for each ISFAQ measure (response delay, feeding interval, settling method) at each age. Table 2 shows the median values used. For response delay, this method allocated parents who reported responding to infant crying in $1 \mathrm{~min}$ or less to an infant-cued subgroup, and parents who took longer than $1 \mathrm{~min}$ to respond to a limit-setting subgroup, at each age. Similarly, parents who reported an interval before feeding of 1 min or less were allocated to an infantcued feeding-interval subgroup, and parents who reported a pre-feed interval longer than 1 min were allocated to a limit-setting subgroup, at each age. For settling method, parents who reported settling when their infant was tired (always, almost always, usually or sometimes) were allocated to an infantcued settling subgroup; parents who reported settling at a regular time (always, almost always, or usually) were assigned to a limit-setting subgroup. For each measure (response delay, feeding interval, settling method) we then cross-tabulated the numbers of parents in each group (GeneralCommunity; Bed-Sharing) who remained above the median, remained below the median, or who changed from above to below (or vice versa), between newborn and $5 \mathrm{~W}$ ages. Potentially, this longitudinal analysis generated four parenting subgroups within each group (General-Community; Bed-Sharing) for each ISFAQ measure (response delay, feeding interval, settling method): stable infant-cued; increasingly infant-cued over age; increasingly limit-setting over age; stable limitsetting.

In step two, objective, video or Diary, methods were used to confirm these group and ISFAQdefined subgroup differences in parenting.

In step three, the parenting groups and subgroups were compared on video, diary and parental questionnaire measures of infant night waking and continuous sleeping at $3 \mathrm{M}$ and $6 \mathrm{M}$ of age. 
Table 2 Diary measures of feed length, feed interval and feed frequency at $2 \mathrm{~W}$ and $5 \mathrm{~W}$ in the groups and general-community subgroups

\begin{tabular}{|c|c|c|c|c|c|c|c|c|c|c|c|c|}
\hline & \multicolumn{6}{|c|}{ Diary $2 \mathrm{~W}$ measures } & \multicolumn{6}{|c|}{ Diary $5 \mathrm{~W}$ measures } \\
\hline & $\begin{array}{l}\text { Night- } \\
\text { time } \\
\text { feed } \\
\text { length }\end{array}$ & $\begin{array}{l}\text { Night-time } \\
\text { feed interval }\end{array}$ & $\begin{array}{l}\text { Night-time } \\
\text { feed } \\
\text { frequency }\end{array}$ & $\begin{array}{l}\text { Day-time } \\
\text { feed } \\
\text { length }\end{array}$ & $\begin{array}{l}\text { Day-time } \\
\text { feed } \\
\text { interval }\end{array}$ & $\begin{array}{l}\text { Day-time } \\
\text { feed } \\
\text { frequency }\end{array}$ & $\begin{array}{l}\text { Night-time } \\
\text { feed length }\end{array}$ & $\begin{array}{l}\text { Night-time } \\
\text { feed interval }\end{array}$ & $\begin{array}{l}\text { Night-time } \\
\text { feed } \\
\text { frequency }\end{array}$ & $\begin{array}{l}\text { Day-time } \\
\text { feed } \\
\text { length }\end{array}$ & $\begin{array}{l}\text { Day-time } \\
\text { feed } \\
\text { interval }\end{array}$ & $\begin{array}{l}\text { Day-time } \\
\text { feed } \\
\text { frequency }\end{array}$ \\
\hline $\begin{array}{l}\text { Bed-Sharing group } \\
(n=19)^{\mathrm{a}}\end{array}$ & $\begin{array}{l}0: 24: 00 \\
(0: 12: 00)\end{array}$ & $\begin{array}{l}1: 37: 00 \\
(0: 35: 00)^{* *}\end{array}$ & $\begin{array}{l}6.58 \\
(2.20) * *\end{array}$ & $\begin{array}{l}0: 19: 00 \\
(0: 10: 00)\end{array}$ & $\begin{array}{l}1: 13: 00 \\
(0: 31: 00)^{* * *}\end{array}$ & $\begin{array}{l}8.28 \\
(2.78)^{* * *}\end{array}$ & $\begin{array}{l}0: 18: 00 \\
(0: 06: 00)\end{array}$ & $\begin{array}{l}1: 47: 00 \\
(0: 25: 00)^{* *}\end{array}$ & $\begin{array}{l}6.25 \\
(1.49)^{* * *}\end{array}$ & $\begin{array}{l}0: 17: 00 \\
(0 \cdot 20 \cdot 00)\end{array}$ & $\begin{array}{l}1: 21: 00 \\
(0 \cdot 26 \cdot 00)^{* *}\end{array}$ & $\begin{array}{l}7.00 \\
(3.26) * *\end{array}$ \\
\hline $\begin{array}{l}\mathrm{GC} \text { group } \\
(n=101)^{\mathrm{a}}\end{array}$ & $\begin{array}{l}0: 23: 00 \\
(0: 11: 00)\end{array}$ & $\begin{array}{l}2: 08: 00 \\
(0: 42: 00)^{* *}\end{array}$ & $\begin{array}{l}5.23 \\
(1.97)^{* *}\end{array}$ & $\begin{array}{l}0: 23: 00 \\
(0: 10: 00)\end{array}$ & $\begin{array}{l}1: 48: 00 \\
(0: 39: 00)^{* * *}\end{array}$ & $\begin{array}{l}5.95 \\
(2.06)^{* * *}\end{array}$ & $\begin{array}{l}0: 22: 00 \\
(0: 09: 00)\end{array}$ & $\begin{array}{l}2: 29: 00 \\
(0: 50: 00) * *\end{array}$ & $\begin{array}{l}4.56 \\
(1.77)^{* * *}\end{array}$ & $\begin{array}{l}0: 20: 00 \\
(0: 12: 00)\end{array}$ & $\begin{array}{l}1: 57: 00 \\
(0: 41: 00)^{* *}\end{array}$ & $\begin{array}{l}5.51 \\
(2.13) * *\end{array}$ \\
\hline \multicolumn{13}{|l|}{$\begin{array}{l}\mathrm{GC} \text { response delay } \\
\text { subgroups }\end{array}$} \\
\hline $\begin{array}{l}\text { 1. Stable } \leqslant 1 \mathrm{~min} \\
(n=35)\end{array}$ & $\begin{array}{l}0: 21: 00 \\
(0: 11: 00)\end{array}$ & $\begin{array}{l}2: 05: 00 \\
(0: 39: 00)\end{array}$ & $\begin{array}{l}5.44 \\
(1.70)\end{array}$ & $\begin{array}{l}0: 19: 00 \\
(0: 08: 00)^{*}\end{array}$ & $\begin{array}{l}1: 41: 00 \\
(0: 34: 00)\end{array}$ & $\begin{array}{l}6.36 \\
(1.91)\end{array}$ & $\begin{array}{l}0: 17: 00 \\
(0: 06: 00)^{* *}\end{array}$ & $\begin{array}{l}2: 18: 00 \\
(0: 41: 00)\end{array}$ & $\begin{array}{l}4.90 \\
(1.61)\end{array}$ & $\begin{array}{l}0: 16: 00 \\
(0: 06: 00)^{* *}\end{array}$ & $\begin{array}{l}1: 48: 00 \\
(0: 35: 00)^{*}\end{array}$ & $\begin{array}{l}6.02 \\
(2.07)\end{array}$ \\
\hline $\begin{array}{l}\text { 2. Changed from }>1 \text { to } \leqslant 1 \mathrm{~min} \\
(n=15)\end{array}$ & $\begin{array}{l}0: 26: 00 \\
(0: 12: 00)\end{array}$ & $\begin{array}{l}1: 50: 00 \\
(0: 34: 00)\end{array}$ & $\begin{array}{l}5.82 \\
(2.21)\end{array}$ & $\begin{array}{l}0: 26: 00 \\
(0: 12: 00)^{*}\end{array}$ & $\begin{array}{l}1: 41: 00 \\
(0: 43: 00)\end{array}$ & $\begin{array}{l}6.55 \\
(2.88)\end{array}$ & $\begin{array}{l}0: 22: 00 \\
(0: 11: 00)^{* *}\end{array}$ & $\begin{array}{l}2: 14: 00 \\
(0: 45: 00)\end{array}$ & $\begin{array}{l}4.99 \\
(1.61)\end{array}$ & $\begin{array}{l}0: 20: 00 \\
(0: 11: 00)^{* *}\end{array}$ & $\begin{array}{l}1: 36: 00 \\
(0: 30: 00)^{*}\end{array}$ & $\begin{array}{l}6.65 \\
(2.22)\end{array}$ \\
\hline $\begin{array}{l}\text { 3. Changed from } \leqslant 1 \text { to }>1 \mathrm{~min} \\
(n=20)\end{array}$ & $\begin{array}{l}0: 25: 00 \\
(0: 12: 00)\end{array}$ & $\begin{array}{l}2: 10: 00 \\
(0: 45: 00)\end{array}$ & $\begin{array}{l}5.27 \\
(2.13)\end{array}$ & $\begin{array}{l}0: 26: 00 \\
(0: 11: 00)^{*}\end{array}$ & $\begin{array}{l}1: 55: 00 \\
(0: 43: 00)\end{array}$ & $\begin{array}{l}5.58 \\
(2.14)\end{array}$ & $\begin{array}{l}0: 25: 00 \\
(0: 09: 00)^{* *}\end{array}$ & $\begin{array}{l}2: 43: 00 \\
(1: 03: 00)\end{array}$ & $\begin{array}{l}4.30 \\
(2.88)\end{array}$ & $\begin{array}{l}0: 25: 00 \\
(0: 10: 00)^{* *}\end{array}$ & $\begin{array}{l}2: 11: 00 \\
(0: 42: 00)^{*}\end{array}$ & $\begin{array}{l}5.12 \\
(1.82)\end{array}$ \\
\hline 4. Stable $>1 \min (n=31)$ & $\begin{array}{l}0: 24: 00 \\
(0: 10: 00)\end{array}$ & $\begin{array}{l}2: 15: 00 \\
(0: 43: 00)\end{array}$ & $\begin{array}{l}4.98 \\
(2.09)\end{array}$ & $\begin{array}{l}0: 25: 00 \\
(0: 10: 00)^{*}\end{array}$ & $\begin{array}{l}1: 53: 00 \\
(0: 42: 00)\end{array}$ & $\begin{array}{l}5.63 \\
(1.57)\end{array}$ & $\begin{array}{l}0: 25: 00 \\
(0: 09: 00)^{* *}\end{array}$ & $\begin{array}{l}2: 34:: 00 \\
(0: 47: 00)\end{array}$ & $\begin{array}{l}4.30 \\
(1.59)\end{array}$ & $\begin{array}{l}0: 23: 00 \\
(0: 08: 00)^{* *}\end{array}$ & $\begin{array}{l}2: 02: 00 \\
(0: 43: 00)^{*}\end{array}$ & $\begin{array}{l}5.55 \\
(2.32)\end{array}$ \\
\hline \multicolumn{13}{|c|}{ GC feeding interval subgroups } \\
\hline 1. Stable $\leqslant 1 \min (n=20)$ & $\begin{array}{l}0: 21: 00 \\
(0: 14: 00)\end{array}$ & $\begin{array}{l}1: 48: 00 \\
(0: 33: 00)^{*}\end{array}$ & $\begin{array}{l}6.08 \\
(1.95)\end{array}$ & $\begin{array}{l}0: 21: 00 \\
(0: 12: 00)\end{array}$ & $\begin{array}{l}1: 32: 00 \\
(0: 28: 00)\end{array}$ & $\begin{array}{l}6.82 \\
(1.93)^{*}\end{array}$ & $\begin{array}{l}0: 18: 00 \\
(0: 06: 00)\end{array}$ & $\begin{array}{l}1: 59: 00 \\
(0: 32: 00)^{* *}\end{array}$ & $\begin{array}{l}5.39 \\
(1.88)^{*}\end{array}$ & $\begin{array}{l}0: 15: 00 \\
(0: 06: 00)^{*}\end{array}$ & $\begin{array}{l}1: 38: 00 \\
(0: 33: 00)\end{array}$ & $\begin{array}{l}6.48 \\
(2.47)\end{array}$ \\
\hline $\begin{array}{l}\text { 2. Changed from }>1 \text { to } \leqslant 1 \mathrm{~min} \\
(n=14)\end{array}$ & $\begin{array}{l}0: 25: 00 \\
(0: 12: 00)\end{array}$ & $\begin{array}{l}1: 58: 00 \\
(0: 45: 00)^{*}\end{array}$ & $\begin{array}{l}5.69 \\
(2.41)\end{array}$ & $\begin{array}{l}0: 24: 00 \\
(0: 13: 00)\end{array}$ & $\begin{array}{l}1: 36: 00 \\
(0: 40: 00)\end{array}$ & $\begin{array}{l}6.86 \\
(3.08)^{*}\end{array}$ & $\begin{array}{l}0: 22: 00 \\
(0: 13: 00)\end{array}$ & $\begin{array}{l}2: 12: 00 \\
(0: 35: 00) * *\end{array}$ & $\begin{array}{l}5.11 \\
(1.40)^{*}\end{array}$ & $\begin{array}{l}0: 20: 00 \\
(0: 12: 00)^{*}\end{array}$ & $\begin{array}{l}1: 50: 00 \\
(0: 34: 00)\end{array}$ & $\begin{array}{l}6.03 \\
(2.37)\end{array}$ \\
\hline $\begin{array}{l}\text { 3. Changed from } \leqslant 1 \text { to }>1 \mathrm{~min} \\
(n=36)\end{array}$ & $\begin{array}{l}0: 25.00 \\
(0: 11: 00)\end{array}$ & $\begin{array}{l}2: 19: 00 \\
(0: 46: 00)^{*}\end{array}$ & $\begin{array}{l}4.89 \\
(1.77)\end{array}$ & $\begin{array}{l}0: 24: 00 \\
(0: 10: 00)\end{array}$ & $\begin{array}{l}1: 58: 00 \\
(0: 47: 00)\end{array}$ & $\begin{array}{l}5.54 \\
(1.92)^{*}\end{array}$ & $\begin{array}{l}0: 24: 00 \\
(0: 09: 00)\end{array}$ & $\begin{array}{l}2: 41: 00 \\
(0: 54: 00)^{* *}\end{array}$ & $\begin{array}{l}4.18 \\
(1.92)^{*}\end{array}$ & $\begin{array}{l}0: 22: 00 \\
(0: 08: 00)^{*}\end{array}$ & $\begin{array}{l}2: 07: 00 \\
(0: 44: 00)\end{array}$ & $\begin{array}{l}5.36 \\
(1.83)\end{array}$ \\
\hline 4. Stable $>1 \min (n=31)$ & $\begin{array}{l}0: 21: 00 \\
(0: 09: 00)\end{array}$ & $\begin{array}{l}2: 12: 00 \\
(0: 36: 00)^{*}\end{array}$ & $\begin{array}{l}5.08 \\
(2.07)\end{array}$ & $\begin{array}{l}0: 24: 00 \\
(0: 08: 00)\end{array}$ & $\begin{array}{l}1: 52: 00 \\
(0: 32: 00)\end{array}$ & $\begin{array}{l}5.51 \\
(1.40)^{*}\end{array}$ & $\begin{array}{l}0: 23: 00 \\
(0: 09: 00)\end{array}$ & $\begin{array}{l}2: 44: 00 \\
(0: 47: 00)^{* *}\end{array}$ & $\begin{array}{l}4.09 \\
(1.53)^{*}\end{array}$ & $\begin{array}{l}0: 23: 00 \\
(0: 09: 00)^{*}\end{array}$ & $\begin{array}{l}2: 01: 00 \\
(0: 36: 00)\end{array}$ & $\begin{array}{l}5.42 \\
(2.04)\end{array}$ \\
\hline \multicolumn{13}{|c|}{ GC settling method subgroups } \\
\hline $\begin{array}{l}\text { 1. Stable 'settled when tired' } \\
(n=31)\end{array}$ & $\begin{array}{l}0: 25: 00 \\
(0: 12: 00)\end{array}$ & $\begin{array}{l}2: 01: 00 \\
(0: 48: 00)\end{array}$ & $\begin{array}{l}5.71 \\
(2.32)\end{array}$ & $\begin{array}{l}0: 24: 00 \\
(0: 11: 00)\end{array}$ & $\begin{array}{l}1: 38: 00 \\
(0: 47: 00)\end{array}$ & $\begin{array}{l}6.52 \\
(2.25)\end{array}$ & $\begin{array}{l}0: 21: 00 \\
(0: 09: 00)\end{array}$ & $\begin{array}{l}2: 10: 00 \\
(0: 49: 00)\end{array}$ & $\begin{array}{l}5.37 \\
(2.12)^{*}\end{array}$ & $\begin{array}{l}0: 19: 00 \\
(0: 09: 00)\end{array}$ & $\begin{array}{l}1: 50: 00 \\
(0: 49: 00)\end{array}$ & $\begin{array}{l}6.13 \\
(2.67)\end{array}$ \\
\hline $\begin{array}{l}\text { 2. Changed from 'settled at } \\
\text { same time' to 'settled when } \\
\text { tired' }(n=21)\end{array}$ & $\begin{array}{l}0: 22: 00 \\
(0: 10: 00)\end{array}$ & $\begin{array}{l}2: 10: 00 \\
(0: 40: 00)\end{array}$ & $\begin{array}{l}5.22 \\
(1.75)\end{array}$ & $\begin{array}{l}0: 23: 00 \\
(0: 10: 00)\end{array}$ & $\begin{array}{l}1: 51: 00 \\
(0: 39: 00)\end{array}$ & $\begin{array}{l}5.76 \\
(1.95)\end{array}$ & $\begin{array}{l}0: 22: 00 \\
(0: 07: 00)\end{array}$ & $\begin{array}{l}2: 29: 00 \\
(0: 45: 00)\end{array}$ & $\begin{array}{l}4.40 \\
(1.43)^{*}\end{array}$ & $\begin{array}{l}0: 22: 00 \\
(0: 10: 00)\end{array}$ & $\begin{array}{l}2: 00: 00 \\
(0: 34: 00)\end{array}$ & $\begin{array}{l}5.36 \\
(1.51)\end{array}$ \\
\hline $\begin{array}{l}\text { 3. Changed from 'settled } \\
\text { when tired' to 'settled at } \\
\text { same time' }(n=16)\end{array}$ & $\begin{array}{l}0: 23: 00 \\
(0: 12: 00)\end{array}$ & $\begin{array}{l}2: 04: 00 \\
(0: 35: 00)\end{array}$ & $\begin{array}{l}5.44 \\
(2.11)\end{array}$ & $\begin{array}{l}0: 23: 00 \\
(0: 10: 00)\end{array}$ & $\begin{array}{l}1: 50: 00 \\
(0: 37: 00)\end{array}$ & $\begin{array}{l}5.89 \\
(1.79)\end{array}$ & $\begin{array}{l}0: 21: 00 \\
(0: 09: 00)\end{array}$ & $\begin{array}{l}2: 40: 00 \\
(1: 03: 00)\end{array}$ & $\begin{array}{l}4.28 \\
(1.96)^{*}\end{array}$ & $\begin{array}{l}0: 21: 00 \\
(0: 08: 00)\end{array}$ & $\begin{array}{l}1: 54: 00 \\
(0: 38: 00)\end{array}$ & $\begin{array}{l}5.85 \\
(2.00)\end{array}$ \\
\hline $\begin{array}{l}\text { 4. Stable 'settled at same } \\
\text { time' }(n=33)\end{array}$ & $\begin{array}{l}0: 24: 00 \\
(0: 11: 00)\end{array}$ & $\begin{array}{l}2: 14: 00 \\
(0: 40: 00)\end{array}$ & $\begin{array}{l}4.86 \\
(1.68)\end{array}$ & $\begin{array}{l}0: 23: 00 \\
(0: 10: 00)\end{array}$ & $\begin{array}{l}1: 54: 00 \\
(0: 32: 00)\end{array}$ & $\begin{array}{l}5.71 \\
(1.97)\end{array}$ & $\begin{array}{l}0: 23: 00 \\
(0: 11: 00)\end{array}$ & $\begin{array}{c}2: 40: 00 \\
(0: 44: 00)\end{array}$ & $\begin{array}{l}4.08 \\
(1.28)^{*}\end{array}$ & $\begin{array}{l}0: 20: 00 \\
(0: 10: 00)\end{array}$ & $\begin{array}{l}2: 01: 00 \\
(0: 40: 00)\end{array}$ & $\begin{array}{l}5.65 \\
(2.03)\end{array}$ \\
\hline
\end{tabular}

Data are mean (SD) frequencies or lengths of time in hours:minutes:seconds.

ANOVA: ${ }^{*} P<0.05 ;{ }^{* *} P<0.01 ;{ }^{* *} P<0.001$.

${ }^{a}$ Group and subgroup sizes given are at $2 \mathrm{~W}$. No $5 \mathrm{~W}$ diary data were obtained from one Bed-Sharing and one General-Community case.

$\mathrm{GC}=$ General-Community. 


\section{Results}

\section{Parental questionnaire (ISFAQ) measures of parenting}

Participants were predominately white, highly educated and married or co-habiting: Table 1 in St James-Roberts et al. (2016) provides detailed figures. The Bed-Sharing group contained fewer firstborn infants. Most General-Community infants (64\%) but all Bed-Sharing infants were still exclusively breast milk fed at 3M. Most (93\%) General-Community mothers planned their infants would usually sleep in cots in their bedroom, but $40-50 \%$ planned to or occasionally did bed-share with their baby for short periods (eg, feeding). All but one Bed-Sharing infant still bed shared through the night at $3 \mathrm{M}$.

\section{Response delay over age}

The longitudinal stability analyses showed that Bed-Sharing parents' reported responsiveness was highly stable, as well as rapid: $95 \%$ planning to and responding to infant cries within a minute at both newborn and 5W ages. Consequently, Bed-Sharing parenting could not in practice be divided into response delay subgroups.

In contrast, $34.3 \%$ of General-Community parents consistently responded to cries within a minute, $30.3 \%$ consistently delayed responding for $>1 \mathrm{~min}$, $15.2 \%$ reduced response delay, and $20.2 \%$ increased response delay, between newborn and $5 \mathrm{~W}$ ages.

\section{Feeding interval over age}

Bed-Sharing parents were again highly stable and infant-cued in their reported parenting, so that $95 \%$ consistently reported feeding within a minute of detecting infant waking, making it impossible, in practice, to divide Bed-Sharing parents into feeding interval subgroups.

Among General-Community parents 20.2\% consistently fed within $1 \mathrm{~min}, 29.8 \%$ consistently implemented a pre-feed interval $>1 \mathrm{~min}$; just $12.8 \%$ reduced the interval to $\leqslant 1 \mathrm{~min}$, and $37.2 \%$ increased the interval from $\leqslant 1$ to $>1$ min with age (the most common of the General-Community group's four parenting subgroups).

\section{Settling methods over age}

Most $(63 \%)$ Bed-Sharing parents planned and implemented settling their infants when tired (always, almost always, usually or sometimes). General-Community parenting was again less stable, so that $30.7 \%$ of these parents reported planning and implementing settling when tired, $32.7 \%$ consistently settling infants to sleep at a regular time, and $36.6 \%$ changed from one to the other (approximately equally in each direction).

These parent-report findings indicate that General-Community parenting was more unstable, as well as more limit-setting, compared with BedSharing parenting. The General-Community group included stable and unstable parenting subgroups.

\section{Video or diary confirmation of the ISFAQ reports}

\section{Response delay}

Table 3 summarises the $5 \mathrm{~W}$ video data. In these recordings, Bed-Sharing infants signalled for a mean of $2 \mathrm{~s}$ before direct parental contact and 10 of 19 Bed-Sharing parents detected waking and intervened before infants signalled. On average, General-Community infants were awake for $3 \mathrm{~min}$ $32 \mathrm{~s}$, and signalled for $1 \mathrm{~min} 3 \mathrm{~s}$, before direct parental contact. These differences, examined in detail in St James-Roberts et al. (2016), are highly statistically significant.

The General-Community subgroups of parents did not differ in how long they took to detect and respond to infant waking at $5 \mathrm{~W}$. However, the video-recorded difference in response delay to infant signalling at $5 \mathrm{~W}$ was significantly different between these subgroups (Table 3). Tukey's honest significant difference (HSD) tests allocated this difference between the four subgroups overall, but the data suggest a binary split: the two parent subgroups who reported delaying response at $5 \mathrm{~W}$ being observed to let infants signal for a mean of around $1.5 \mathrm{~min}$, compared with around $0.5 \mathrm{~min}$ for the two subgroups who reported responding within a minute (Table 3 ). These findings support the parents' questionnaire reports and the mediansplit method.

\section{Feeding interval}

Consistently implemented intervals before feeding would be expected to lengthen the spacing between feeds. Because most GeneralCommunity infants were removed from the video 
Table 3 Video measures of night-time parenting behaviours at $5 \mathrm{~W}$ in the groups and General-Community subgroups

\begin{tabular}{|c|c|c|c|c|c|c|c|c|c|c|}
\hline & \multicolumn{6}{|c|}{ Video: infant behaviour when settled at bed-time } & \multicolumn{4}{|c|}{ Video: parenting contact behaviours } \\
\hline & $\begin{array}{l}\% \\
\text { Infants } \\
\text { asleep }\end{array}$ & $\begin{array}{l}\% \\
\text { Infants } \\
\text { drowsy }\end{array}$ & $\begin{array}{l}\% \\
\text { Infants } \\
\text { awake } \\
\text { content }\end{array}$ & $\begin{array}{l}\% \text { Infants } \\
\text { distressed }\end{array}$ & $\begin{array}{l}\% \\
\text { Infants } \\
\text { feeding }\end{array}$ & $\begin{array}{l}\% \text { Infants } \\
\text { indeterminate }\end{array}$ & $\begin{array}{l}\text { Mean (SD) interval } \\
\text { between infant } \\
\text { waking and direct } \\
\text { parental contact }\end{array}$ & $\begin{array}{l}\text { Mean (SD) interval } \\
\text { between infant } \\
\text { signalling and } \\
\text { direct parental } \\
\text { contact }\end{array}$ & $\begin{array}{l}\text { Mean (SD) \% of } \\
\text { waking periods } \\
\text { including direct } \\
\text { parental contact }\end{array}$ & $\begin{array}{l}\text { Mean (SD) \% of } \\
\text { sleeping periods } \\
\text { including direct } \\
\text { parental contact }\end{array}$ \\
\hline Bed-Sharing group $(n=18)^{(\mathrm{c})}$ & $50.0^{(\mathrm{a})}$ & $00.0^{(\mathrm{a})}$ & $11.1^{(\mathrm{a})}$ & $11.1^{(\mathrm{a})}$ & $22.2^{(\mathrm{a})}$ & $5.6^{(\mathrm{a})}$ & $0: 00: 14(0: 00: 40)^{* * *}$ & $0: 00: 02(0: 00: 08)^{* *}$ & $89.03(26.10)^{* * *}$ & $76.16(00.06)^{* * *}$ \\
\hline $\begin{array}{l}\mathrm{GC} \text { group }(n=99)^{(\mathrm{c})} \\
\mathrm{GC} \text { response delay } \\
\text { subgroups }\end{array}$ & $49.5^{(\mathrm{a})}$ & $11.1^{\text {(a) }}$ & $24.2^{(\mathrm{a})}$ & $5.1^{(\mathrm{a})}$ & $00.0^{(\mathrm{a})}$ & $10.1^{(\mathrm{a})}$ & $0: 03: 32(0: 03: 13)^{* * *}$ & $0: 01: 03(0: 01: 33)^{* *}$ & $26.70(28.11)^{* * *}$ & $06.2(0.21)^{* * *}$ \\
\hline 1. Stable $\leqslant 1 \min (n=34)$ & 58.8 & 11.8 & 11.8 & 05.9 & 00.0 & 11.8 & 0:02:49 (0:03:03) & 0:00:31 (0:00:54)* & $32.2(31.1)$ & $10.1(26.5)$ \\
\hline $\begin{array}{l}\text { 2. Changed from }>1 \text { to } \leqslant 1 \mathrm{~min} \\
(n=15)\end{array}$ & 40.0 & 00.0 & 46.7 & 06.7 & 00.0 & 06.7 & $0: 03: 44(0: 03: 22)$ & $0: 00: 31(0: 00: 26)^{*}$ & $25.0(26.8)$ & $00.6(00.9)$ \\
\hline $\begin{array}{l}\text { 3. Changed from } \leqslant 1 \text { to }>1 \mathrm{~min} \\
(n=20)\end{array}$ & 70.0 & 00.0 & 15.0 & 00.0 & 00.0 & 15.0 & 0:03:49 (0:03:39) & $0: 01: 34(0: 02: 27)^{*}$ & $23.5(27.6)$ & $07.7(24.0)$ \\
\hline $\begin{array}{l}\text { 4. Stable }>1 \min (n=30) \\
\text { GC feeding interval } \\
\text { subgroups }\end{array}$ & 32.1 & 25.0 & 28.6 & 07.1 & 00.0 & 07.1 & 0:03:54 (0:02:58) & $0: 01: 33(0: 01: 31)^{*}$ & $22.8(25.2)$ & $03.9(18.8)$ \\
\hline 1. Stable $\leqslant 1 \min (n=20)$ & 73.7 & 05.3 & 10.5 & 00.0 & 00.0 & 10.5 & 0:03:13 (0:03:01) & 0:00:39 (0:01:09) & $35.4(35.3)$ & $13.3(31.2)$ \\
\hline $\begin{array}{l}\text { 2. Changed from }>1 \text { to } \leqslant 1 \mathrm{~min} \\
(n=14)\end{array}$ & 33.3 & 00.0 & 50.0 & 08.3 & 00.0 & 08.3 & 0:04:17 (0:04:13) & 0:01:02 (0:02:03) & $35.1(31.5)$ & $07.0(23.3)$ \\
\hline $\begin{array}{l}\text { 3. Changed from } \leqslant 1 \text { to }>1 \mathrm{~min} \\
(n=36)\end{array}$ & 52.6 & 12.1 & 15.2 & 09.1 & 00.0 & 06.1 & 0:03:05 (0:03:07) & 0:01:18 (0:02:04) & $23.3(25.8)$ & $07.4(24.5)$ \\
\hline $\begin{array}{l}\text { 4. Stable }>1 \text { min }(n=29) \\
\text { GC settling method } \\
\text { subgroups }\end{array}$ & 32.1 & 17.9 & 28.6 & 03.6 & 00.0 & 17.9 & 0:04:09 (0:03:03) & 0:01:01 (0:00:57) & $19.5(21.3)$ & $00.6(01.0)$ \\
\hline $\begin{array}{l}\text { 1. Stable 'settled when tired' } \\
(n=31)\end{array}$ & $58.1^{(\mathrm{b})}$ & $16.1^{(b)}$ & $19.4^{(\mathrm{b})}$ & $03.2^{(\mathrm{b})}$ & $00.00^{(b)}$ & $03.2^{(\mathrm{b})}$ & $0: 02: 45(0: 02: 25)$ & 0:00:34 (0:00:31) & $22.2(27.7)$ & $09.5(28.1)$ \\
\hline $\begin{array}{l}\text { 2. Changed from 'settled at } \\
\text { same time' to 'settled when } \\
\text { tired' }(n=20)\end{array}$ & $47.6^{(\mathrm{b})}$ & $09.5^{(\mathrm{b})}$ & $14.3^{(\mathrm{b})}$ & $09.5^{(\mathrm{b})}$ & $00.00^{(b)}$ & $19.0^{(\mathrm{b})}$ & 0:03:51 (0:05:05) & 0:01:21 (0:01:28) & $35.1(34.1)$ & $06.7(20.8)$ \\
\hline $\begin{array}{l}\text { 3. Changed from 'settled } \\
\text { when tired' to 'settled at } \\
\text { same time' }(n=17)\end{array}$ & $56.3^{(\mathbf{b})}$ & $00.0^{(\mathrm{b})}$ & $12.5^{(\mathbf{b})}$ & $00.0^{(\mathrm{b})}$ & $00.0^{(\mathrm{b})}$ & $31.3^{(\mathrm{b})}$ & 0:02:51 (0:02:59) & $0: 01: 30(0: 02: 24)$ & $26.5(26.2)$ & $03.2(11.4)$ \\
\hline $\begin{array}{l}\text { 4. Stable' settled at same } \\
\text { time' }(n=31)\end{array}$ & $38.7^{(\mathrm{b})}$ & $12.9^{(\mathrm{b})}$ & $41.9^{(\mathrm{b})}$ & $06.5^{(\mathrm{b})}$ & $00.0^{(\mathrm{b})}$ & $00.0^{(\mathrm{b})}$ & 0:04:41 (0:04:01) & 0:01:07 (0:01:44) & $25.6(24.9)$ & $03.9(17.9)$ \\
\hline \multicolumn{11}{|c|}{$\begin{array}{l}\text { Data are percentages or lengths of time in hours:minutes:seconds. } \\
\text { ANOVA }{ }^{*} P<0.05 ;{ }^{* *} P<0.01 ;{ }^{* *} P<0.001{ }^{(\mathrm{a})} P \text { Pearson } \chi^{2} P<0.001 ;{ }^{(\mathrm{b})} P \text { earson } \chi^{2} P=0.013 \text {. } \\
\text { (c) No video data at } 5 \mathrm{~W} \text { were obtained for two General-Community and one Bed-Sharing case. } \\
3 \mathrm{M}=\text { three months; } 6 \mathrm{M}=\text { six months; } \mathrm{GC}=\text { General-Community; } 5 \mathrm{~W}=\text { five weeks. }\end{array}$} \\
\hline
\end{tabular}


recording for feeding at 5W (only nine of 99 were recorded feeding), diary data were used to measure these inter-feed intervals (the time between the end of one and start of the next feed). Table 2 presents the findings. General-Community infants had longer inter-feed intervals, and fewer feeds, than Bed-Sharing infants in the day and night at $2 \mathrm{~W}$ and $5 \mathrm{~W}$.

The General-Community feeding interval subgroups differed in night-time, but not day-time, inter-feed intervals at both $2 \mathrm{~W}$ and $5 \mathrm{~W}$ (Table 2). Tukey's HSD tests at $5 \mathrm{~W}$ identified shorter nighttime feed intervals in the subgroup who planned and implemented feeding within 1 min than in groups who planned and implemented, or implemented, pre-feed intervals $>1 \mathrm{~min} \quad(P<0.05)$. These subgroup differences in night-time interfeed interval were substantial, averaging $>20 \mathrm{~min}$ at $2 \mathrm{~W}$ and $>40 \mathrm{~min}$ at $5 \mathrm{~W}$ (Table 2 ). These findings support the parents' questionnaire reports and the median-split method.

\section{Settling method}

Settling at a scheduled time should reduce the number of infants already asleep or feeding when parents settled them for the night. As Table 3 shows, around half the infants in both groups were already asleep when video recording began. However, General-Community infants were more likely to be drowsy or awake content, and less likely to be feeding, when settled than Bed-Sharing infants.

Among the General-Community subgroups, infants who were consistently settled at a regular time appear more likely to have been awake content when settled than infants in the other subgroups (Table 3 ). Even so, nearly $40 \%$ of these infants were already asleep when settled for the night by parents.

These video findings provide some support for the parent-reported night-time settling method differences, but these group and subgroup differences were less robust than the differences in response delay and feeding interval.

\section{Relationships between response delay, feeding interval and settling method}

The concepts of infant-cued and limit-setting parenting imply a degree of overlap in parenting response delay, feeding interval and settling method. That is the case when the GeneralCommunity and Bed-Sharing groups are compared (Tables 2 and 3). Except for feed length, the groups differed substantially in all the Diary and video measures of all three parenting components.

In contrast, the General-Community response delay, feeding interval, and settling method subgroups were largely distinct in their video- and diary-measured parenting characteristics at $2 \mathrm{~W}$ and $5 \mathrm{~W}$. An exception is that parents who planned and implemented rapid responding had shorter day-time feeds at $2 \mathrm{~W}$, and shorter day and nighttime feeds and day-time feeding intervals at $5 \mathrm{~W}$ (Table 2). Tukey's HSD tests confirmed that this was mainly because the General-Community subgroup of parents who consistently responded rapidly also fed for shorter periods than both subgroups who delayed responding $(P<0.05)$. This was not matched by significantly more frequent feeding, but both General-Community subgroups who responded rapidly at $5 \mathrm{~W}$ tended to have short inter-feed intervals (Table 2). The GeneralCommunity subgroup who consistently settled infants when tired had more frequent night-time feeds at $5 \mathrm{~W}$ than other subgroups (Table 2).

These overlaps in General-Community subgroup parenting are of a type consistent with the distinction between infant-cued and limit-setting parenting. However, the subgroup differences in Diary and video-measured parenting are much greater than their overlap, so that separate subgroup comparisons on outcome measures of infant sleep-waking are worthwhile.

\section{Hypothesis testing: comparing the parenting groups and subgroups on infant sleep-waking at $3 M$ and $6 M$ of age}

Exact agreement between methods was not expected because of criterion and measurement differences. For instance, Diaries measured infants across three successive nights, defined as $7 \mathrm{pm}$ to 7am, while videos measured a single night defined by parents (around 9-10 h from 10pm). Nevertheless, group and subgroup differences should be similar across methods.

Bed-Sharing infants had more frequent, shorter sleep periods and woke more often during the night than General-Community infants on all video, Diary and questionnaire measures (Table 4). 
Among the General-Community subgroups, delayed responding predicted longer night-time sleep periods in 3M Diary, but not in video or parental questionnaire measures, or in $6 \mathrm{M}$ measures (Table 4), providing weak support for our primary hypothesis.

Our secondary hypothesis predicted that use of feeding intervals in the early weeks would lead to longer day-time feeds at $5 \mathrm{~W}$ to compensate for the longer feeding intervals and fewer feeds at night. As Table 3 shows, the figures support that expectation. At $5 \mathrm{~W}$, each day-time feed of the subgroup who consistently implemented a pre-feeding interval lasted 8 min longer, on average, than the feeds of the subgroup who consistently minimised night-time pre-feeding intervals (Tukey's HSD test difference $P<0.05$ ).

Stably implemented feeding intervals also predicted the infant outcome sleep-waking measures from all three methods at $3 \mathrm{M}$, and both questionnaire measures at $6 \mathrm{M}$ age (Table 4$)$. Figure 1 shows the number of infants in the Bed-Sharing group, and General-Community feeding interval subgroups, who remained asleep for periods of $\geqslant 5 \mathrm{~h}$ at night at $3 \mathrm{M}$ measured by video, Diary and questionnaire methods. Like Bed-Sharing infants, only $\sim 40 \%$ of General-Community infants who were fed within a minute at both newborn and $5 \mathrm{~W}$ ages were asleep for continuous night-time periods of $5 \mathrm{~h}$ or more at $3 \mathrm{M}$. In comparison, infants whose parents planned and implemented an interval before feeding were around twice as likely to remain asleep for $\geqslant 5 \mathrm{~h}$ night-time periods, at $3 \mathrm{M}$.

The parents' bed-time settling method did not predict $3 \mathrm{M}$ infant sleep-waking on any measure (Table 4). The prediction of questionnairereported sleep periods lasting $\geqslant 5 \mathrm{~h}$ at $6 \mathrm{M}$ may be a chance finding but, in any case, settling method did not predict infant sleep-waking robustly.

Stepwise logistic regressions were used to examine whether the feeding interval predictions in Figure 1 were affected by the response delay scores (see Table 5). The feeding interval predictions all remained highly significant and were mostly unaffected by adding or removing the response delay scores. The exception was that adding response delay improved the prediction of Diary-measured sleep periods lasting $5 \mathrm{~h}$ or more at $3 \mathrm{M}$. However, the effect size was small and, when entered in the first step, the response delay scores alone failed to predict any of the measures of 3M infant sleeping (Table 5).

\section{Possible confounders}

First and later-born infants did not differ in $3 \mathrm{M}$ or 6M sleep-waking, while the consistency of the evidence across methods, groups and subgroups rules out the group difference in video indeterminate time, described in St James-Roberts et al. (2016), as an explanation of the findings. Analyses reported in St James-Roberts et al. (2015) found that $66 \%$ of infants exclusively fed breast milk, and $62.5 \%$ of infants fed formula or mixed breast and formula milk at $3 \mathrm{M}$, were settled for $\geqslant 5 \mathrm{~h}$ nighttime periods at $3 \mathrm{M}$, which is not significantly different.

\section{Discussion}

There is evidence that RCTs of parenting programmes can be confounded by parents' unwillingness to implement interventions that conflict with their values or circumstances [Medical Research Council (MRC), 2007; Olds et al., 2007]. Accordingly, following MRC (2007) guidelines, this study was designed to complement existing RCT evidence by using video recording and other methods to observe London infants' and parents' typical night-time behaviours in their normal home environments. Assessments focussed on the first $3 \mathrm{M}$ of age: the period when most western infants develop prolonged sleep periods at night. A General-Community group of 101 infants and parents, most of whom were expected to adopt limitsetting parenting methods, was compared with a group of 19 planned Bed-Sharing parents and babies, who were expected to adopt highly infantcued parenting methods. This comparison group included parents who intended to bed-share from before their baby's birth and did so consistently, unlike 'reactive' bed-sharers who respond to their infant's night waking and signalling by switching to bed sharing (Germo et al., 2007).

The first finding was that around a third of London General-Community parents did not implement their planned form of parenting, or changed from an infant-cued towards a limitsetting strategy, or vice versa, in the first five postnatal weeks. This variability, initially 


\begin{tabular}{|c|c|c|c|c|c|}
\hline & \multicolumn{3}{|l|}{$3 \mathrm{M}$} & \multicolumn{2}{|l|}{$6 \mathrm{M}$} \\
\hline & $\begin{array}{l}\text { Video: mean (SD) } \\
\text { length of infant } \\
\text { night-time sleep } \\
\text { periods (hours:minutes: } \\
\text { seconds) }\end{array}$ & $\begin{array}{l}\text { Diary: mean (SD) } \\
\text { length of infant } \\
\text { night-time sleep } \\
\text { periods (hours: } \\
\text { minutes) }\end{array}$ & $\begin{array}{l}\text { Questionnaire: } \\
\text { mean (SD) } \\
\text { no. of times } \\
\text { infant woke } \\
\text { per night }\end{array}$ & $\begin{array}{l}\text { Questionnaire: mean } \\
\text { (SD) no. of nights } \\
\text { infants remained } \\
\text { asleep for } \geqslant 5 \mathrm{~h} \\
\text { periods }\end{array}$ & $\begin{array}{l}\text { Questionnaire: } \\
\text { mean (SD) no. } \\
\text { of times infant } \\
\text { woke per night }\end{array}$ \\
\hline & $1: 59: 26(1: 00: 40)^{* *}$ & $2: 04(0: 46)^{* *}$ & $2.45(0.78)^{* *}$ & $2.94(2.96)^{* *}$ & $3.14(2.21)^{* * *}$ \\
\hline $\begin{array}{l}\mathrm{GC} \text { group }(n=101)^{\mathrm{a}} \\
\mathrm{GC} \text { response delay subgroups }\end{array}$ & $3: 33: 28(2: 32: 42)^{* *}$ & $3: 33(1: 56)^{* *}$ & $1.59(1.13)^{* *}$ & $5.19(2.45)^{* *}$ & $1.30(1.13)^{* * *}$ \\
\hline $\begin{array}{l}\text { 1. } \leqslant 1 \text { min both newborn and } 5 \mathrm{~W} \text { ages } \\
(n=34)\end{array}$ & $3: 38: 00(2: 49: 59)$ & $3: 07(1: 27)^{*}$ & $1.90(1.40)$ & $4.41(2.79)$ & $1.56(1.46)$ \\
\hline 2. Changed from $>1$ to $\leqslant 1 \min (n=15)$ & $2: 54: 35(1: 16: 17)$ & $3: 06(1: 11)^{*}$ & $1.33(0.90)$ & $5.57(2.38)$ & $1.25(1.10)$ \\
\hline 3. Changed from $\leqslant 1$ to $>1 \min (n=21)$ & 4:42:52 (3:21:41) & $4: 42(2: 58)^{*}$ & $1.27(0.99)$ & $5.83(2.12)$ & $0.92(0.94)$ \\
\hline $\begin{array}{l}\text { 4. }>1 \text { min both newborn and } 5 \mathrm{~W} \text { ages } \\
(n=31)\end{array}$ & 3:03:22 (1:51:49) & $3: 24(1: 28)^{*}$ & $1.62(0.97)$ & $5.37(2.20)$ & $1.28(0.80)$ \\
\hline \multicolumn{6}{|l|}{ GC feeding interval subgroups } \\
\hline $\begin{array}{l}1 . \leqslant 1 \mathrm{~min} \text { both newborn and } 5 \mathrm{~W} \text { ages } \\
(n=20)\end{array}$ & $2: 35: 58(1: 37: 57)^{* *}$ & $2: 41(1: 03)^{*}$ & $2.53(1.45)^{* * *}$ & $3.83(2.83)^{*}$ & $2.19(1.44)^{* *}$ \\
\hline 2. Changed from $>1$ to $\leqslant 1 \min (n=14)$ & $2: 10: 28(1: 13: 47)^{* *}$ & $2: 54(1: 04)^{*}$ & $1.81(1.29)^{* * *}$ & $4.73(2.87)^{*}$ & $1.59(1.16)^{* *}$ \\
\hline \multirow{2}{*}{$\begin{array}{l}\text { 3. Changed from } \leqslant 1 \text { to }>1 \text { min }(n=37) \\
\text { 4. }>1 \text { min both newborn and } 5 \mathrm{~W} \text { ages } \\
(n=30)\end{array}$} & $4: 42: 03(3: 24: 24)^{* *}$ & $4: 07(2: 30)^{*}$ & $1.27(0.99) * * *$ & $5.93(2.08)^{*}$ & $0.77(0.97)^{* *}$ \\
\hline & $3: 30: 17(1: 39: 26)^{* *}$ & $3: 53(1: 55)^{*}$ & $1.20(0.57)^{* * *}$ & $5.63(1.92)^{*}$ & $1.13(0.71)^{* *}$ \\
\hline $\begin{array}{l}\text { 1. 'Settled when tired' both newborn } \\
\text { and } 5 \mathrm{~W} \text { ages }(n=31)\end{array}$ & 3:28:51 (2:09:58) & $3: 12(1: 13)$ & $1.56(1.05)$ & $4.86(2.63)^{*}$ & $1.66(1.24)$ \\
\hline $\begin{array}{l}\text { 2. Changed from 'settled same time' } \\
\text { to 'settled when tired' }(n=21)\end{array}$ & $3: 34: 16(2: 37: 44)$ & $3: 10(1: 45)$ & $1.93(1.37)$ & $4.10(2.69)^{*}$ & $1.45(1.00)$ \\
\hline $\begin{array}{l}\text { 3. Changed from 'settled when tired' } \\
\text { to 'settled at same time' }(n=16)\end{array}$ & 3:42:45 (3:17:12) & $3: 42(2: 14)$ & $1.66(1.27)$ & $5.50(2.34)^{*}$ & $1.00(0.76)$ \\
\hline $\begin{array}{l}\text { 4. 'Settled at same time' both newborn } \\
\text { and } 5 \mathrm{~W} \text { ages }(n=33)\end{array}$ & $3: 32: 48(2: 32: 18)$ & $4: 02(2: 22)$ & $1.37(0.96)$ & $6.06(1.88)^{*}$ & $1.00(0.91)$ \\
\hline
\end{tabular}

Data are numbers or lengths of time in hours:minutes:seconds.

ANOVA: ${ }^{*} P<0.05 ;{ }^{*} P<0.01 ;{ }^{* *} P<0.001$.

a Group and subgroup sizes given are at $3 \mathrm{M}$. At $6 \mathrm{M}$, eight General-Community and one Bed-Sharing case did not return data.

$3 \mathrm{M}=$ three months; $6 \mathrm{M}=$ six months; $\mathrm{GC}=$ General-Community; $5 \mathrm{~W}=$ five weeks 


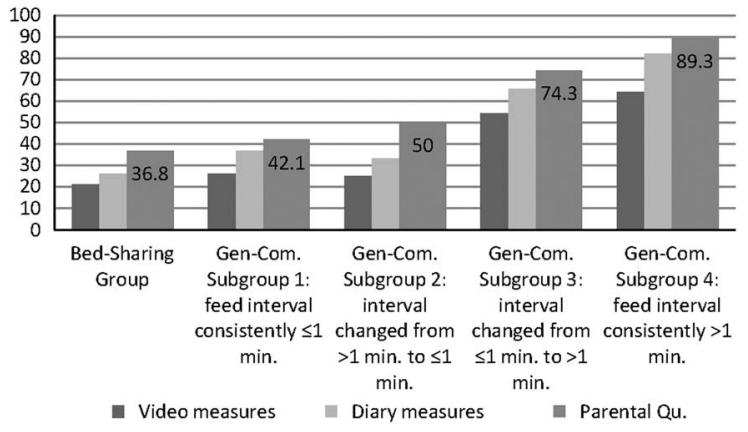

Figure 1 Percentages of infants in the Bed-Sharing group and each General-Community feed interval subgroup who remained asleep for $\geqslant 5 \mathrm{~h}$ periods at night at three months of age: video, diary and questionnaire measures

Table 5 Results of stepwise logistic regressions using feed interval to predict the video, Diary and questionnaire measures of the infant $\geqslant 5 \mathrm{~h}$ sleep periods at $3 \mathrm{M}$ included in Figure $1^{\mathrm{a}}$

\begin{tabular}{|c|c|c|c|}
\hline & $\begin{array}{l}\chi^{2} \text { for } \\
\text { step }\end{array}$ & $\begin{array}{l}P \text { for } \\
\text { step }\end{array}$ & $\begin{array}{l}\text { Nagelkerke } R^{2} \\
\text { for model }\end{array}$ \\
\hline \multicolumn{4}{|l|}{ Video measures } \\
\hline Feed interval & 14.1 & $<0.001$ & 0.20 \\
\hline Response delay & 0.12 & 0.73 & 0.20 \\
\hline \multicolumn{4}{|l|}{ Diary measures } \\
\hline Feed interval & 9.05 & 0.003 & 0.12 \\
\hline Response delay & 5.02 & 0.025 & 0.19 \\
\hline \multicolumn{4}{|l|}{$\begin{array}{l}\text { Questionnaire } \\
\text { measures }\end{array}$} \\
\hline Feed interval & 13.0 & $<0.001$ & 0.18 \\
\hline Response delay & 3.08 & 0.08 & 0.21 \\
\hline
\end{tabular}

${ }^{\text {a }}$ Feed interval was entered in the first step and response delay added in the second step.

The $\chi^{2}$ figure gives the goodness of fit for the step; the Nagelkerke $R^{2}$ estimates the effect size.

Entering response delay in the first step did not predict the infant sleep measures significantly.

described by Goodlin-Jones et al. (2001), stood in marked contrast to the stability in infant-cued parenting shown by the Bed-Sharing group. These parenting inconsistencies are important in their own right. For instance, some parents may manage the infant crying peak at one to two months of age (Barr, 1990) by increasing responsiveness, so that parenting is reactive in such cases. The implication is that research is needed to understand why parents change care strategies and whether anticipatory guidance can help to prevent unintended consequences, such as reactive bed sharing.
This finding also has implications for RCT design. Even recent RCTs of parenting programmes for preventing infant sleep problems have provided little evidence that parents consistently implement the intended interventions or differ from control groups (Stremler et al., 2013; Hiscock et al., 2014). RCTs need to document implementation and reasons for implantation failure.

The second finding was that stable limit-setting parenting led to longer infant night-time sleep periods at $3 \mathrm{M}$ and $6 \mathrm{M}$ of age. Analyses of the videos confirm that this involved increased infant sleep length over age, not variations in parents' detection of infant waking (St James-Roberts et al., 2015). Supporting our secondary, but not primary, hypothesis one parenting strategy - consistently implementing a short interval before feeding - led to fewer night-time feeds, longer night-time feed intervals, and longer day-time feeds at $5 \mathrm{~W}$, and twice as many infants sleeping continuously for $5 \mathrm{~h}$ or more at night at $3 \mathrm{M}$, compared with immediate or rapid feeding. A proviso is that $11 \%$ of infants were not asleep for $\geqslant 5 \mathrm{~h}$ periods at night at $3 \mathrm{M}$ even where parents consistently implemented an interval before feeding. This is in keeping with transactional models which posit that infant, as well as parenting, factors influence infant sleep-waking development (Sadeh et al., 2010). The nature of these infant factors requires further research.

These findings need to be interpreted in light of the methodological limitations of this study, including that participants were highly educated, largely white parents in stable relationships in London, the small size of the comparison group and General-Community subgroups, and reliance on only three 24-h diary days at each of three ages and one night of video recording at each of two ages. The use of median splits to divide the General-Community group into subgroups at each age has disadvantages, but these concern the likelihood that analyses will fail to identify subgroup differences. In contrast, our video and diary findings confirmed the validity of our median-split analyses, while the finding of highly significant differences in the group and General-Community subgroup measures of infant sleep-walking across video, diary and questionnaire methods at $3 \mathrm{M}$ of age, and at both $3 \mathrm{M}$ and $6 \mathrm{M}$ ages, is highly unlikely to be due to error or chance.

As others point out (Tikotzky et al., 2015) a crucial limitation of the longitudinal observation 
method is that it cannot prove causal relationships between study variables. It follows that factors other than feeding interval, including infant characteristics, may be responsible for the infant outcomes observed. Although cross-lagged and other statistical methods could be used to explore causality, they too have limited power to resolve this question.

Although we accept this limitation, four RCTs the most powerful research method for establishing causation - have provided replicated evidence that limit-setting parenting increases the proportion of infants who have long night-time sleep periods by 3M of age (Wolfson et al., 1992; Pinilla and Birch, 1993; St James-Roberts et al., 2001; Symon et al., 2005). Mirroring findings here, one RCT found that parents did implement feeding intervals (St JamesRoberts et al., 2001) and another that limit-setting parenting, including feeding intervals, reduced night-time feed frequency and increased day-time feeds at four to six weeks, and night-time sleep periods at 3M of age (Pinilla and Birch, 1993). The current study's value lies in complementing these RCT findings by adding evidence that these parenting features are associated with the development of prolonged infant night-time sleeping in representative home environments during the period when most western infants become settled at night. Our findings are also consistent with observational evidence that infants in general increase their daytime feeds at around two months of age (Wright, 1993) and that infants whose mothers nurse them less at bedtime show a steeper increase in night-time sleep (Philbrook and Teti, 2016). In addition, they complement RCT evidence that limit-setting interventions are particularly effective where infants feed frequently (Nikolopoulou and St James-Roberts, 2003; Hiscock et al., 2014). In this study, increasing the interval before feeding as infants aged was the General-Community parents' most common longitudinal parenting strategy, suggesting that parents who implemented this from birth adopted a form of parenting which is normative as infants grow older. Evidence that parenting typically becomes less infant-cued as infants age has been found, too, in other English (Williams et al., 2016) and Norwegian (Sudnes and Andenaes, 2016) studies. It seems likely that parents' initial concern about their baby's well-being and weight gain gives way to a more deliberated approach as infants develop.

Taken together, these findings provide substantial, but not conclusive, evidence that a short interval before feeding leads to a cascade of adaptations in infant self-regulation and sleepwaking which many infants manage successfully by $3 \mathrm{M}$ of age. RCT confirmation of this finding is needed, but physiological research to establish how feed spacing can alter infant metabolic selfregulation may be a useful prior step. For instance, an interval before feeding may increase wakefulness and feeding vigour, resulting in greater intake and physiological adjustments which extend sleep length and the interval before the next feed. The existing findings do not support the long-standing assumption that breast milk constituents require 3M-old infants to wake frequently at night.

For clinical purposes, any benefits which stem from introducing an interval before feeding need to be balanced against its disadvantages, including the evidence that this leads to increased distress in General-Community infants, relative to infants whose parents use highly infant-cued parenting including bed sharing. However, no significant differences in infant night-time distress were found between General-Community subgroups with minimal versus typical pre-feeding intervals ( $\mathrm{St}$ James-Roberts et al., 2016). Moreover, the difference in distress between General-Community and Bed-Sharing groups was larger at $2 \mathrm{~W}(\sim 30 \mathrm{~min}$ per night) than at five or 12 weeks of age $(\sim 12-13 \mathrm{~min}$ per night). While some parents may consider that any infant distress should be avoided, others may judge that a night-time increase of around $1 \mathrm{~min} / \mathrm{h}$ after $5 \mathrm{~W}$ of age is justified by the potential benefits of implementing a short interval before feeding.

These findings can be conveyed to parents and professionals to help them to make informed choices but, in our view, should not be incorporated prescriptively into health service recommendations. Instead, the findings should provide the basis for further research to substantiate, and refine, their use. The existing evidence implies that limit-setting parenting should result in less infant night-time distress and improved sleeping over the long-term, while improved sleeping should help to support healthy infant development (El-Sheikh and Sadeh 2015). However, there is little direct evidence to confirm these overall benefits, or those of infant-cued parenting.

'Authoritative' parenting, that combines warmth with limit-setting, is especially effective in supporting older children's development (Pratt et al., 1988). Perhaps because of contemporary 
concerns with 'baby-friendly' parenting and maintaining breast-feeding, the question of how and when parenting can support infant sleep-waking and other aspects of development has received sparse attention. Instead of perpetuating the debate about the 'best' form of parenting, the question of when to transition from infant-cued to limit-setting parenting so as to maintain breastfeeding, support infant self-regulation, and prevent long-term night waking and distress, seems likely to be a fruitful focus for research.

\section{Acknowledgements}

The authors thank the Wellcome Trust, the families who participated, the staff of Queen Charlotte's and Chelsea Hospital for their support, and Clara Heath for assistance with data processing.

\section{Financial Support}

This research was funded by Wellcome Trust Research Project Grant No. 85752.

\section{Conflicts of Interest}

None.

\section{References}

Anders, T.F., Halpern, L.F. and Hua, J. 1992: Sleeping through the night: a developmental perspective. Pediatrics 90, 554-60.

Anders, T.F. and Keener, M. 1985: Developmental course of nighttime sleep-wake patterns in full-term and premature infants during the first year of life. Sleep 8, 173-92.

Ball, H.L. 2007: Bed-Sharing practices of initially breastfed infants in the first 6 months of life. Infant and Child Development 16, 387-401.

Barr, R.G. 1990: The normal crying curve: what do we really know? (Annotation). Developmental Medicine and Child Neurology 32, 356-62.

Barr, R.G., Kramer, M.S., Boisjoly, C., Mcvey-White, L. and Pless, I.B. 1988: Parental diary of infant cry and fuss behavior. Archives of Disease in Childhood 63, 380-87.

Barr, R.G., Paterson, J., Macmartin, L., Lehtonen, L. and Young, S. 2005: Prolonged and unsoothable crying bouts in infants with and without colic. Journal of Developmental and Behavioral Pediatrics 26, 14-22.

Douglas, P. and Hill, P. 2013: Behavioral sleep interventions in the first six months of life do not improve outcomes for mothers or infants: a systematic review. Journal of Developmental and Behavioral Pediatrics 34, 497-507.
El-Sheikh, M. and Sadeh, A. 2015: Sleep and development: advancing theory and research. Monographs of the Society for Child Development 80, 1-215.

Germo, G.R., Chang, E.S., Keller, M.A. and Goldberg, W.A. 2007: Child sleep arrangements and family life: perspectives from mothers and fathers. Infant and Child Development 16, 433-56.

Goodlin-Jones, B.L., Burnham, M., Gaylor, E. and Anders, T.M. 2001: Night waking, sleep-wake organisation, and selfsoothing in the first year of life. Journal of Developmental and Behavioral Pediatrics 22, 226-32.

Henderson, J.M., France, K.G., Owens, J.L. and Blampied, N.M. 2010: Sleeping through the night: the consolidation of selfregulated sleep across the first year of life. Pediatrics 126, e1081-1087.

Hiscock, H., Cook, F., Bayer, J., Le, H.N., Mensah, F., Cann, W., Symon, B. and St James-Roberts, I. 2014: Preventing early infant sleep and crying problems and postnatal depression: a randomized trial. Pediatrics 133, e346-354.

Hysing, M., Harvey, A.G., Torgersen, L., Ystrom, E., Reichborn-Kjennerud, T. and Sivertsen, B. 2014: Trajectories and predictors of nocturnal awakenings and sleep duration in infants. Journal of Developmental and Behavioral Pediatrics 35, 309-16.

IBM 2013. SPSS version 22. New York, NY: IBM.

Jenni, O.G. and O'Connor, B.B. 2005: Children's sleep: an interplay between culture and biology. Pediatrics 115, 204-16.

Mindell, J.A., Sadeh, A., Wiegand, B., How, T.H. and Goh, D.Y.T. 2010: Cross-cultural differences in infant and toddler sleep. Sleep Medicine 11, 274-80.

Moore, T. and Ucko, L. 1957: Night waking in early infancy: part 1. Archives of Disease in Childhood 32, 333-42.

Morrell, J. 1999: The role of maternal cognitions in infant sleep problems as assessed by a new instrument, the maternal cognitions about infant sleep questionnaire. Journal of Child Psychology and Psychiatry 40, 247-58.

Medical Research Council (MRC) 2007: MRC guidelines for developing and evaluating complex interventions: new guidance. Retrieved 15 February 2015 from http://www.mrc. ac.uk/Utilities/Documentrecord/index.htm?d=MRC004871.

Nikolopoulou, M. and St James-Roberts, I. 2003: Preventing sleeping problems in infants who are at risk of them. Archives of Disease in Childhood 88, 108-11.

Olds, D.L., Sadler, L. and Kitzman, H. 2007: Programs for parents of infants and toddlers: recent evidence from randomised trials. Journal of Child Psychology and Psychiatry 48, 355-91.

Philbrook, L.E. and Teti, D.M. 2016: Bidirectional associations between bedtime parenting and infant sleep: parenting quality, parenting practices, and their interaction. Journal of Family Psychology 30, 431-41.

Pinilla, T. and Birch, L. 1993: Help me make it through the night: behavioral entrainment of breast-fed infants' sleep patterns. Pediatrics 91, 436-44.

Pratt, M.W., Kerig, P., Cowan, P.A. and Cowan, C.P. 1988: Mothers and fathers teaching 3-year-olds: authoritative 
parenting and adult scaffolding of young children's learning. Developmental Psychology 24, 832-39.

Sadeh, A. 2004: Brief screening questionnaire for infant sleep problems: validation and findings for an internet sample. Pediatrics 113, e-570-577.

Sadeh, A. and Sivan, Y. 2009: Clinical practice: sleep problems during infancy. European Journal of Pediatrics 168, 1159-164.

Sadeh, A., Tikotzky, L. and Scher, A. 2010: Parenting and infant sleep. Sleep Medicine Reviews 14, 89-96.

Sitnick, S.L., Goodlin-Jones, B.L. and Anders, T.F. 2008: The use of actigraphy to study sleep disorders in preschoolers: some concerns about detection of nighttime awakenings. Sleep 31, 395-401.

St James-Roberts, I., Alvarez, M., Csipke, E., Abramsky, T., Goodwin, J. and Sorgenfrei, E. 2006: Infant crying and sleeping in London, Copenhagen and when parents adopt a "proximal" form of care. Pediatrics 117, e1146-1155.

St James-Roberts, I., Roberts, M., Hovish, K. and Owen, C. 2015: Video evidence that London infants can resettle themselves back to sleep after waking in the night, as well as sleep for long periods, by 3 months of age. Journal of Developmental and Behavioral Pediatrics 36, 324-29.

St James-Roberts, I., Roberts, M., Hovish, K. and Owen, C. 2016: Descriptive figures for differences in parenting and infant night-time distress in the first three months of age. 17, 611-21.

St James-Roberts, I., Sleep, J., Morris, S., Owen, C. and Gillham, P. 2001: Use of a behavioral programme in the first 3 months to prevent infant crying and sleep problems. Journal of Paediatrics and Child Health 37, 289-97.

Stremler, R., Hodnett, E., Kenton, L., Lee, K., Weiss, S., Weston, J. and Willan, A. 2013: Effect of a behavioraleducational intervention on sleep for primiparous women and their infants in early postpartum: multisite randomised controlled trial. British Medical Journal 346, f1164.

Sudnes, A. and Andenaes, A. 2016: Parental regulation of infant sleep: round-the-clock efforts for social synchronization. Infant Mental Health Journal 37, 247-58.

Symon, B.G., Marley, J.E., Martin, J.A. and Norman, E.R. 2005: Effect of a consultation teaching behavior modification on sleep performance in infants: randomised controlled trial. Medical Journal of Australia 182, 215-18.

Tikotzky, L. and Sadeh, A. 2009: Maternal sleep-related cognitions and infant sleep: a longitudinal study from pregnancy through the 1st year. Child Development 80, 860-74.

Tikotzky, L., Sadeh, A., Volovich, E., Manber, R., Meiri, G. and Shahar, G. 2015: Infant Sleep development from 3 to 6 months postpartum: links with maternal sleep and parental involvement. In El-Sheikh, M. and Sadeh, A., editors, Sleep and development: advancing theory and research. Monographs of the Society for Research in Child Development, Serial No. 316, Vol 80 No.1. 107-124, Wiley, Oxford U.K.

Williams, C.J., Kessler, D., Fernyhough, C., Lewis, G. and Pearson, R.M. 2016: The association between maternalreported responses to infant crying at 4 weeks and 6 months and offspring depression at 18: a longitudinal study. Archives of Women's Mental Health 19, 401-8.

Wolfson, A., Lacks, P. and Futterman, A. 1992: Effects of parent training on infant sleeping patterns, parents' stress and perceived parental competence. Journal of Consulting and Clinical Psychology 60, 41-48.

Wright, P. 1993: Mothers' ideas about feeding in early infancy. In St James-Roberts, I., Harris, G. and Messer, D., editors. Infant crying, feeding and sleeping: development, problems and treatments. Hemel Hemstead: Harvester Wheatsheaf, 99-117. 